\title{
On the Condition of a Matrix Arising in the Numerical Inversion of the Laplace Transform
}

\section{By Walter Gautschi}

\begin{abstract}
Bellman, Kalaba, and Lockett recently proposed a numerical method for inverting the Laplace transform. The method consists in first reducing the infinite interval of integration to a finite one by a preliminary substitution of variables, and then employing an $n$-point Gauss-Legendre quadrature formula to reduce the inversion problem (approximately) to that of solving a system of $n$ linear algebraic equations. Luke suggests the possibility of using Gauss-Jacobi quadrature (with parameters $\alpha$ and $\beta$ ) in place of Gauss-Legendre quadrature, and in particular raises the question whether a judicious choice of the parameters $\alpha, \beta$ may have a beneficial influence on the condition of the linear system of equations. The object of this note is to investigate the condition number cond $(n, \alpha, \beta)$ of this system as a function of $n, \alpha$, and $\beta$. It is found that cond $(n, \alpha, \beta)$ is usually larger than cond $(n, \beta, \alpha)$ if $\beta>\alpha$, at least asymptotically as $n \rightarrow \infty$. Lower bounds for cond $(n, \alpha, \beta)$ are obtained together with their asymptotic behavior as $n \rightarrow \infty$. Sharper bounds are derived in the special cases $\alpha=\beta, n$ odd, and $\alpha=\beta= \pm \frac{1}{2}, n$ arbitrary. There is also a short table of cond $(n, \alpha, \beta)$ for $\alpha, \beta=-.8(.2) 0, .5,1,2,4,8,16, \beta \leqq \alpha$, and $n=5,10,20,40$. The general conclusion is that cond $(n, \alpha, \beta)$ grows at a rate which is something like a constant times $(3+\sqrt{ } 8)^{n}$, where the constant depends on $\alpha$ and $\beta$, varies relatively slowly as a function of $\alpha, \beta$, and appears to be smallest near $\alpha=\beta=-1$. For quadrature rules with equidistant points the condition grows like $(2 \sqrt{ } 2 / 3 \pi) 8^{n}$.
\end{abstract}

1. In [4], Bellman, Kalaba, and Lockett propose a numerical procedure to invert the Laplace transform

$$
\int_{0}^{\infty} e^{-s t} u(t) d t=F(s)
$$

Briefly, the procedure consists of first substituting $x=e^{-t}$, to bring (1.1) into the form

$$
\int_{0}^{1} x^{s-1} g(x) d x=F(s), \quad g(x)=u(-\ln x),
$$

and then employing Gaussian quadrature to approximate (1.2) by

$$
\sum_{i=1}^{n} w_{i} x_{i}{ }^{k} g\left(x_{i}\right)=F(k+1), \quad(k=0,1,2, \cdots, n-1),
$$

where $x_{i}$ are the zeros of the shifted Legendre polynomical $p_{n}(x)=P_{n}(2 x-1)$ and $w_{i}$ the associated weight factors. Letting $y_{i}=w_{i} g\left(x_{i}\right)$, the method thus boils down to solving the system of linear algebraic equations

Received May 9, 1968. 


$$
\sum_{i=1}^{n} x_{i}{ }^{k} y_{i}=F(k+1), \quad(k=0,1,2, \cdots, n-1) .
$$

In reviewing the work of Bellman et al., Y. L. Luke [8] generalizes their approach by employing the substitution $x=e^{-v t}(v>0)$ in (1.1), and by using Jacobi polynomials in place of Legendre polynomials. This again leads to a system of equations (1.4) where now $x_{i}$ are the zeros of the shifted Jacobi polynomial $p_{n}{ }^{(\alpha, \beta)}(x)$ $=P_{n}{ }^{(\alpha, \beta)}(2 x-1)$, and $F(k+1)$ on the right must be replaced by $F((k+1) v)$.

The system (1.4) can be solved analytically in a number of ways, the coefficient matrix being a Vandermonde matrix. However, as noted in [4], the ill-conditioned character of the system may well require high-precision calculations, especially if $n$ is fairly large. Luke [8] raises the question of whether or not "the detrimental effects of ill-conditioning can be removed or mitigated by the use of other choices of $\alpha$ and $\beta$ " (other than $\alpha=\beta=0$ ). The purpose of this note is to give a detailed answer to this question.

We first obtain a closed expression for the condition number of the coefficient matrix in (1.4). In Section 3 we compare the condition number for $p_{n}{ }^{(\alpha, \beta)}$ with that for $p_{n}{ }^{(\beta, \alpha)}$ and find that the former is usually larger than the latter if $\beta>\alpha$, at least asymptotically as $n \rightarrow \infty$. Section 4 contains a short table of the condition number for $p_{n}^{(\alpha, \beta)}$, where $\alpha, \beta=-.8(.2) 0, .5,1,2,4,8,16, \beta \leqq \alpha$, and $n=5,10,20,40$. Section 5 exhibits lower bounds for the condition number, together with their asymptotic behavior. Sharper results are obtained in Section 6 in the case $\alpha=\beta$, $n$ odd, and in Section 7 for general $n$, and $\alpha=\beta= \pm \frac{1}{2}$. For comparison we consider in Section 8 the case of equidistant abscissas $x_{i}$.

The general conclusion is that the condition number grows at a rate which is something like a constant times $(3+\sqrt{ } 8)^{n}\left[(2 \sqrt{ } 2 / 3 \pi) 8^{n}\right.$ for equidistant abscissas], where the constant depends on $\alpha$ and $\beta$ and varies relatively slowly as a function of $\alpha$ and $\beta$. As expected, there is no escape from ill-conditioning, which, after all, only reflects the fact that the original inversion problem (1.1) is not well posed (cf., in this connection, [1], [2], [3], [9], [11], [13], [14]).

2. Let $p_{n}(x)$ be an arbitrary polynomial of degree $n$ whose zeros $x_{i}$ are distinct and located in the interval $[0,1]$. Let

$$
V\left(p_{n}\right)=\left[\begin{array}{cccc}
1 & 1 & \cdots & 1 \\
x_{1} & x_{2} & \cdots & x_{n} \\
\cdots \cdots & \cdots & \cdots & \cdots \\
x_{1}^{n-1} & x_{2}^{n-1} & \cdots & x_{n}{ }^{n-1}
\end{array}\right]
$$

denote the Vandermonde matrix of the zeros $x_{i}$. We shall consider the condition number

$$
\operatorname{cond}_{\infty}\left[V\left(p_{n}\right)\right]=\left\|V\left(p_{n}\right)\right\|_{\infty}\left\|\left[V\left(p_{n}\right)\right]^{-1}\right\|_{\infty},
$$

where $\|\cdot\|_{\infty}$ denotes the $\infty$-matrix norm ("maximum row sum"). Clearly,

$$
\left\|V\left(p_{n}\right)\right\|_{\infty}=n .
$$

In [5] we have shown that under the assumptions made,

$$
\left\|\left[V\left(p_{n}\right)\right]^{-1}\right\|_{\infty}=\max _{i} \prod_{j \neq i}\left(\frac{1+x_{j}}{\left|x_{i}-x_{j}\right|}\right) .
$$


Combining (2.3) and (2.4), and rewriting (2.4) in terms of $p_{n}$ and its derivative, we obtain

$$
\operatorname{cond}_{\infty}\left[V\left(p_{n}\right)\right]=\frac{n\left|p_{n}(-1)\right|}{\min _{i}\left\{\left(1+x_{i}\right)\left|p_{n}{ }^{\prime}\left(x_{i}\right)\right|\right\}}
$$

3. We now let $p_{n}$ be the shifted Jacobi polynomial $p_{n}{ }^{(\alpha, \beta)}(x)=P_{n}^{(\alpha, \beta)}(2 x-1)$, $\alpha>-1, \beta>-1$. We first show that

$$
\operatorname{cond}_{\infty}\left[V\left(p_{n}{ }^{(\alpha, \beta)}\right)\right]=\gamma_{n} \frac{P_{n}^{(\beta, \alpha)}(3)}{P_{n}^{(\alpha, \beta)}(3)} \operatorname{cond}_{\infty}\left[V\left(p_{n}^{(\beta, \alpha)}\right)\right], \quad \frac{1}{2}<\gamma_{n}<2,
$$

where the constant $\gamma_{n}$ depends on $\alpha$ and $\beta$. Indeed, it is well known that

$$
\begin{aligned}
p_{n}{ }^{(\alpha, \beta)}(x) & =(-1)^{n} p_{n}{ }^{(\beta, \alpha)}(1-x), \\
\left.p_{n}{ }^{(\alpha, \beta)}\right)^{\prime}(x) & =(-1)^{n+1} p_{n}{ }^{(\beta, \alpha)^{\prime}}(1-x) .
\end{aligned}
$$

In particular, if $x_{i}$ is a zero of $p_{n}^{(\alpha, \beta)}$ then $\xi_{i}=1-x_{i}$ is a zero of $p_{n}{ }^{(\beta, \alpha)}$. Therefore,

$$
\begin{aligned}
\left(1+x_{i}\right)\left|p_{n}{ }^{(\alpha, \beta)^{\prime}}\left(x_{i}\right)\right| & =\left(1+x_{i}\right)\left|p_{n}{ }^{(\beta, \alpha)^{\prime}}\left(\xi_{i}\right)\right| \\
& =\frac{1+x_{i}}{2-x_{i}}\left(1+\xi_{i}\right)\left|p_{n}^{(\beta, \alpha)^{\prime}}\left(\xi_{i}\right)\right|,
\end{aligned}
$$

and since $\frac{1}{2}<(1+x) /(2-x)<2$ for $0<x<1$, it follows that

$$
\min _{i}\left\{\left(1+x_{i}\right)\left|p_{n}{ }^{(\alpha, \beta)^{\prime}}\left(x_{i}\right)\right|\right\}=\frac{1}{\gamma_{n}} \min _{i}\left\{\left(1+\xi_{i}\right)\left|p_{n}{ }^{(\beta, \alpha)^{\prime}}\left(\xi_{i}\right)\right|\right\}, \frac{1}{2}<\gamma_{n}<2 .
$$

Consequently, by (2.5),

$$
\operatorname{cond}_{\infty}\left[V\left(p_{n}{ }^{(\alpha, \beta)}\right)\right]=\gamma_{n}\left|p_{n}{ }^{(\alpha, \beta)}(-1) / p_{n}{ }^{(\beta, \alpha)}(-1)\right| \operatorname{cond}_{\infty}\left[V\left(p_{n}{ }^{(\beta, \alpha)}\right)\right],
$$

which is equivalent to (3.1).

Noting that $[10$, p. 194]

$$
P_{n}^{(\beta, \alpha)}(3) \sim \frac{n^{-1 / 2}}{\pi^{1 / 2} 2^{(2 \alpha+5) / 4}}(3+\sqrt{ } 8)^{n+(\alpha+\beta+1) / 2} \quad(n \rightarrow \infty),
$$

we obtain from (3.1),

$$
\operatorname{cond}_{\infty}\left[V\left(p_{n}{ }^{(\alpha, \beta)}\right)\right] \sim \gamma_{n} \cdot 2^{(\beta-\alpha) / 2} \operatorname{cond}_{\infty}\left[V\left(p_{n}^{(\beta, \alpha)}\right)\right], \quad(n \rightarrow \infty) .
$$

Our computations (cf. Section 4 ) have revealed that in most cases the minimum in (2.5) is assumed for $x_{i}$ near $\frac{1}{2}$ (though not necessarily closest to $\frac{1}{2}$ ), so that in these cases $\gamma_{n} \approx 1$. Taking this into account it appears from (3.4) that for $n$ sufficiently large the condition number for $p_{n}{ }^{(\alpha, \beta)}$ is greater than that for $p_{n}{ }^{(\beta, \alpha)}$ if $\beta>\alpha$. As was observed by computation this remains generally true for smaller values of $n$ as well (typically for those of Table 1), although in a few instances in the region $-1<\alpha, \beta<0, \beta>\alpha$, it was found that $\operatorname{cond}_{\infty}\left[V\left(p_{n}{ }^{(\alpha, \beta)}\right)\right]$ is slightly less than $\operatorname{cond}_{\infty}\left[V\left(p_{n}{ }^{(\beta, \alpha)}\right)\right]$.

4. In order to compute the condition number in $(2.5)$ for $p_{n}(x)=p_{n}{ }^{(\alpha, \beta)}(x)$, we make use of the fact that these polynomials satisfy the orthogonality relation 


$$
\int_{0}^{1} p_{n}(x) p_{m}(x)(1-x)^{\alpha} x^{\beta} d x=h_{n} \delta_{n, m}
$$

where $h_{n}=\Gamma(n+\alpha+1) \Gamma(n+\beta+1) /((2 n+\alpha+\beta+1) n ! \Gamma(n+\alpha+\beta+1))$ and $\delta_{n, m}$ is the Kronecker delta. With $p_{r}^{*}(x)=h_{r}{ }^{-1 / 2} p_{r}(x)$ denoting the normalized polynomials, we may compute $p_{n}^{*}(x)$ from the recurrence relation

$$
\begin{aligned}
& p_{r+1}^{*}(x)=\left(\left(x-a_{r}\right) p_{r}^{*}(x)-b_{r} p_{r-1}^{*}(x)\right) / b_{r+1}, \quad(r=0,1,2, \cdots, n-1), \\
& p_{-1}^{*}(x)=0, \quad p_{0}^{*}(x)=\left\{\frac{\Gamma(\alpha+\beta+2)}{\Gamma(\alpha+1) \Gamma(\beta+1)}\right\}^{1 / 2} .
\end{aligned}
$$

where

$$
\begin{aligned}
& a_{0}=\frac{1}{2}\left(1-\frac{\alpha-\beta}{\alpha+\beta+2}\right), \\
& a_{r}=\frac{1}{2}\left\{1-\frac{\alpha^{2}-\beta^{2}}{(2 r+\alpha+\beta)(2 r+\alpha+\beta+2)}\right\}, \quad(r \geqq 1), \\
& b_{1}=\frac{1}{\alpha+\beta+2}\left\{\frac{(\alpha+1)(\beta+1)}{\alpha+\beta+3}\right\}^{1 / 2}, \\
& b_{r}=\frac{1}{2 r+\alpha+\beta}\left\{\frac{r(r+\alpha)(r+\beta)(r+\alpha+\beta)}{(2 r+\alpha+\beta-1)(2 r+\alpha+\beta+1)}\right\}^{1 / 2}, \quad(r \geqq 2) .
\end{aligned}
$$

The zeros of $p_{n}{ }^{*}(x)$ may now be computed from (4.2) by a combination of Newton's method and successive deflation as described in $[6$, p. 261]. Hence the condition number of $V\left(p_{n}\right)$ can be computed directly from (2.5) for any value of $\alpha$ and $\beta$. Selected results* are shown in Table 1. (The numbers in parentheses denote the powers of 10 by which the preceding numbers are to be multiplied.) For reasons indicated at the end of Section 3 we restrict our tabulation to the region $\beta \leqq \alpha$.

The results in Table 1 indicate that $\operatorname{cond}_{\infty}\left[V\left(p_{n}{ }^{(\alpha, \beta)}\right)\right]$ for fixed $\alpha$ is an increasing function of $\beta$, if $-1<\alpha \leqq 0$, and changes from a decreasing to an increasing function as $\beta$ varies from -1 to $\alpha$, if $\alpha>0$. There is thus a "valley" of low condition number extending approximately (and more or less independently of $n$ ) along the line $\beta=-1+2 \alpha / 7$, as was determined by additional calculations. Along this valley, as well as along the diagonal $\alpha=\beta$, and near the line $\beta=-1$, the condition number increases with $\alpha$ and thus appears to be smallest near $\alpha=\beta=-1$.

5. A lower bound for the condition number in (2.5) may be obtained as follows. Let

$$
\max _{0 \leqq x \leqq 1}\left|p_{n}(x)\right|=\mu_{n} .
$$

* In the range $-1<\alpha \leqq 3,-1<\beta \leqq 3, \beta \leqq \alpha$, and for $n=5$ and $n=8$, the zeros of $p_{n}{ }^{(\alpha, \beta)}(x)$ as computed were checked against those tabulated in [7]. Disagreement never exceeded one unit of the last (eighth) significant digit. For $n=40$, successive deflation was used only for the first 20 zeros. The remaining zeros were obtained from the original polynomial by Newton's method and a simple search procedure. 


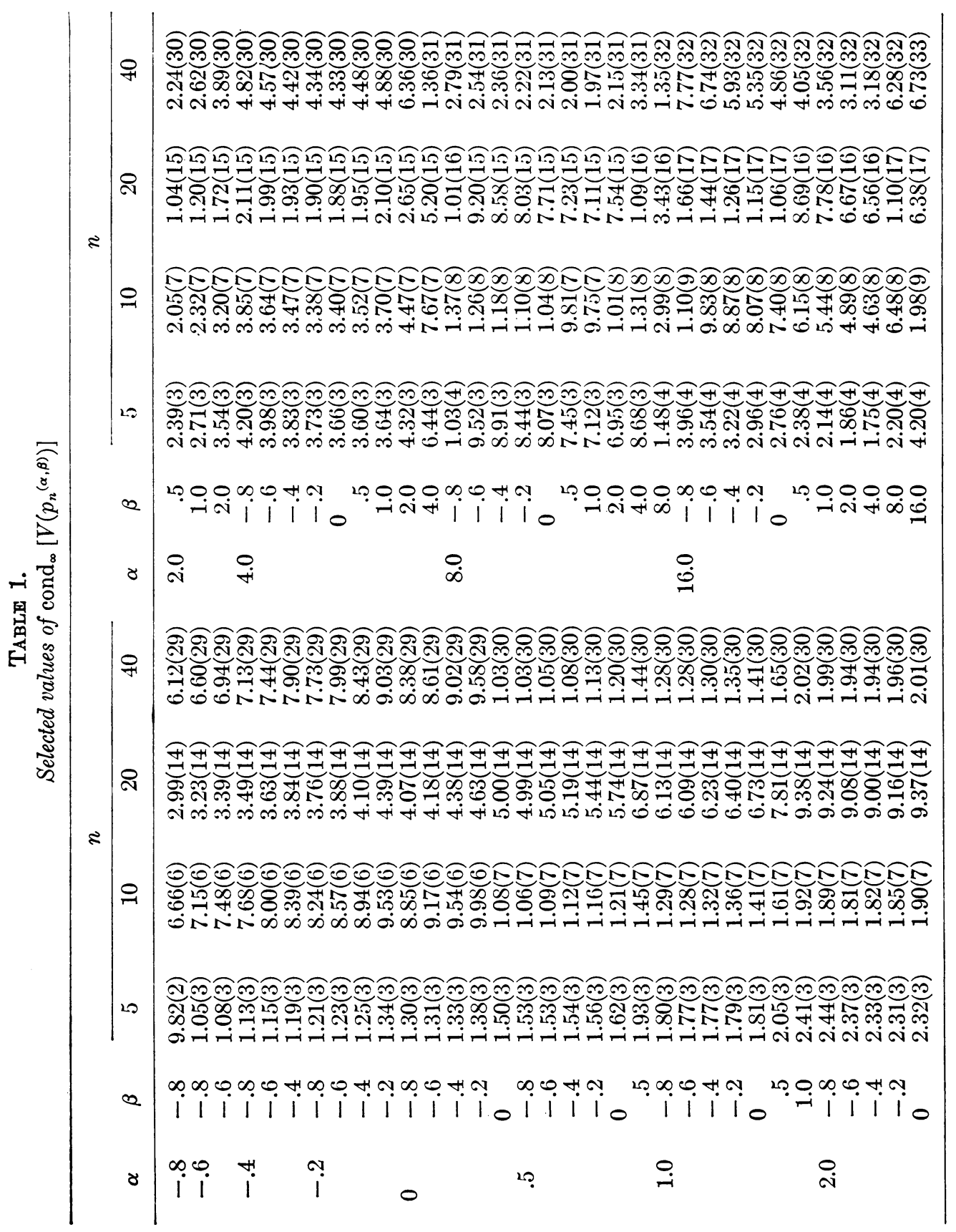

Since $\left|\mu_{n}{ }^{-1} p_{n}(x)\right| \leqq 1$ on $[0,1]$ it follows from a theorem of Markov (see, e.g., [12, p. 36]) that $\left|\mu_{n}^{-1} p_{n}{ }^{\prime}(x)\right| \leqq 2 n^{2}$ on $[0,1]$, and so 


$$
\left(1+x_{i}\right)\left|p_{n}{ }^{\prime}\left(x_{i}\right)\right| \leqq 4 n^{2} \mu_{n}, \quad(i=1,2, \cdots, n) .
$$

Consequently, by (2.5),

$$
\operatorname{cond}_{\infty}\left[V\left(p_{n}\right)\right] \geqq \kappa_{n}, \quad \kappa_{n}=\left|p_{n}(-1)\right| / 4 n \mu_{n} .
$$

If $p_{n}(x)=p_{n}{ }^{(\alpha, \beta)}(x)$, we may take advantage of known asymptotic results for Jacobi polynomials to obtain an asymptotic expression for $\kappa_{n}$ in (5.3). As $n \rightarrow \infty$, we have $[10$, p. 380]

$$
\begin{aligned}
\mu_{n} & \sim n^{q} / \Gamma(q+1) \text { if } q \geqq-\frac{1}{2} \\
& \sim \pi^{-1 / 2}\left|\alpha+\frac{1}{2}\right|^{-\alpha / 2-1 / 4}\left|\beta+\frac{1}{2}\right|^{-\beta / 2-1 / 4}|\alpha+\beta+1|^{(\alpha+\beta+1) / 2} n^{-1 / 2} \\
& \text { if }-1<q<-\frac{1}{2},
\end{aligned}
$$

where $q=\max (\alpha, \beta)$. Combining (5.4) with (3.3) we obtain from (5.3)

$$
\kappa_{n} \sim \frac{\Gamma(q+1)}{\sqrt{ } \pi 2^{(2 \alpha+13) / 4}} n^{-(q+3 / 2)}(3+\sqrt{ } 8)^{n+(\alpha+\beta+1) / 2}, \quad\left(q \geqq-\frac{1}{2}, n \rightarrow \infty\right),
$$

and

$$
\begin{aligned}
\kappa_{n} \sim \frac{\left|\alpha+\frac{1}{2}\right|^{\alpha / 2+1 / 4}\left|\beta+\frac{1}{2}\right|^{\beta / 2+1 / 4}}{2^{(2 \alpha+13) / 4}|\alpha+\beta+1|^{(\alpha+\beta+1) / 2}} n^{-1}(3+\sqrt{ } 8)^{n+(\alpha+\beta+1) / 2}, & \\
& \left(-1<q<-\frac{1}{2}, n \rightarrow \infty\right) .
\end{aligned}
$$

The powers of $n$ appearing in (5.5), (5.6) are due to the crudeness of the inequality (5.2) and do not reflect the true asymptotic behavior of $\operatorname{cond}_{\infty}\left[V\left(p_{n}{ }^{(\alpha, \beta)}\right)\right]$. In fact, if $x_{i}$ is restricted to a closed interval in the interior of $[0,1]$ (e.g., $i$ such that $x_{i}$ is the smallest zero of $p_{n}{ }^{(\alpha, \beta)}$ larger than or equal to $\left.\frac{1}{2}\right)$, then it is known [10, p. 237] that

$$
\left|p_{n}{ }^{(\alpha, \beta)^{\prime}}\left(x_{i}\right)\right| \dot{\sim} n^{1 / 2}, \quad(n \rightarrow \infty),
$$

the symbol $\dot{\sim}$ meaning that the ratio of the left-hand and right-hand expression remains between certain positive bounds depending only on $\alpha$ and $\beta$. It thus follows from (2.5) and (3.3) that

$$
\operatorname{cond}_{\infty}\left[V\left(p_{n}{ }^{(\alpha, \beta)}\right)\right] \geqq \kappa_{n}{ }^{\prime}, \quad \kappa_{n}{ }^{\prime} \dot{\sim}(3+\sqrt{ } 8)^{n}, \quad(n \rightarrow \infty) .
$$

If, as all numerical evidence indicates, the points at which the minimum in (2.5) is assumed remain in a closed interval inside the open interval $(0,1)$ as $n \rightarrow \infty$, then inequality in (5.8) may be replaced by equality.

6. Considerably sharper bounds can be had if $\alpha=\beta$. We thus consider

$$
\begin{aligned}
p_{n}(x)=C_{n}{ }^{(\sigma)}(2 x-1)=\frac{\Gamma\left(\sigma+\frac{1}{2}\right) \Gamma(n+2 \sigma)}{\Gamma(2 \sigma) \Gamma\left(n+\sigma+\frac{1}{2}\right)} P_{n}^{(\sigma-1 / 2, \sigma-1 / 2)}(2 x-1, & \\
\sigma & >-\frac{1}{2},
\end{aligned}
$$

and for convenience we assume that $n$ is odd. Then, by symmetry, $x_{i}=\frac{1}{2}$ for some $i=i_{0}$, so that for this zero,

$$
p_{n}{ }^{\prime}\left(x_{i_{0}}\right)=p_{n}{ }^{\prime}\left(\frac{1}{2}\right)=2 C_{n}{ }^{(\sigma)^{\prime}}(0)=2(n+2 \sigma-1) C_{n-1}^{(\sigma)}(0) .
$$

Since 


$$
\begin{aligned}
C_{n-1}^{(\sigma)}(0) & =(-1)^{(n-1) / 2} \frac{\Gamma((n+2 \sigma-1) / 2)}{\Gamma(\sigma) \Gamma((n+1) / 2)}, \quad(\sigma \neq 0) \\
& =2(-1)^{(n-1) / 2} /(n-1), \quad(\sigma=0),
\end{aligned}
$$

we obtain

$$
\begin{aligned}
\left(1+x_{i_{0}}\right)\left|p_{n}{ }^{\prime}\left(x_{i_{0}}\right)\right| & =6 \frac{\Gamma((n+2 \sigma+1) / 2)}{|\Gamma(\sigma)| \Gamma((n+1) / 2)}, \quad(\sigma \neq 0) \\
& =6, \quad(\sigma=0) .
\end{aligned}
$$

Hence, from (2.5),

$$
\begin{aligned}
\operatorname{cond}_{\infty}\left[V\left(p_{n}\right)\right] \geqq \kappa_{n}, \quad \kappa_{n} & =\frac{n}{6}\left|C_{n}{ }^{(\sigma)}(3)\right| \frac{|\Gamma(\sigma)| \Gamma((n+1) / 2)}{\Gamma((n+2 \sigma+1) / 2)}, \quad(\sigma \neq 0) \\
& =\frac{n}{6} C_{n}{ }^{(0)}(3), \quad(\sigma=0) .
\end{aligned}
$$

From the known asymptotic behavior of $P_{n}^{(\sigma-1 / 2, \sigma-1 / 2)}(x)$ as $n \rightarrow \infty[10$, p. 194] and from Stirling's formula we find

$$
C_{n}^{(\sigma)}(3) \sim \frac{\Gamma\left(\sigma+\frac{1}{2}\right)}{\sqrt{ } \pi \Gamma(2 \sigma) 2^{(\sigma+2) / 2}} n^{\sigma-1}(3+\sqrt{ } 8)^{n+\sigma}, \quad(\sigma \neq 0, n \rightarrow \infty) .
$$

Furthermore,

$$
C_{n}{ }^{(0)}(3)=\frac{2}{n} T_{n}(3) \sim \frac{1}{n}(3+\sqrt{ } 8)^{n}, \quad(n \rightarrow \infty),
$$

where $T_{n}(x)$ is the Chebyshev polynomial of the first kind. Substituting in (6.2), and using Stirling's formula and the duplication formula for the gamma function, we obtain

$$
\kappa_{n} \sim \frac{1}{6 \cdot 8^{\sigma / 2}}(3+\sqrt{ } 8)^{n+\sigma}, \quad\left(\sigma>-\frac{1}{2}, n \rightarrow \infty\right)
$$

a result which obviously improves upon (5.5), (5.6) and is more precise than (5.8).

The case $p_{n}(x)=P_{n}(2 x-1)$ originally considered in [4] corresponds to $\sigma=\frac{1}{2}$, in which case (6.4) gives

$$
\kappa_{n} \sim \frac{1}{6 \cdot 8^{i}}(3+\sqrt{ } 8)^{n+1 / 2}, \quad\left(\sigma=\frac{1}{2}, n \rightarrow \infty\right) .
$$

The corresponding analysis for even $n$ appears to be rather more difficult, for general $\sigma>-\frac{1}{2}$, and we shall not pursue this any further. If $\sigma=0$, or $\sigma=1$, then (6.4) remains valid for general $n$, as will be seen in the next section.

7. The cases $\alpha=\beta= \pm \frac{1}{2}$ merit special attention since the Jacobi polynomials then reduce to Chebyshev polynomials (of the first and second kind), the zeros and weight factors of which are known explicitly.

We begin with $\alpha=\beta=-\frac{1}{2}$, or, equivalently $p_{n}(x)=T_{n}(2 x-1)$. We have

$$
\left|p_{n}(-1)\right|=T_{n}(3)=\frac{1}{2}\left[(3+\sqrt{ } 8)^{n}+(3-\sqrt{ } 8)^{n}\right],
$$




$$
\left|p_{n}(-1)\right|>\frac{1}{2}(3+\sqrt{ } 8)^{n} .
$$

Since the zeros $x_{i}$ of $p_{n}(x)$ satisfy

$$
2 x_{i}-1=\cos \theta_{i}, \quad \theta_{i}=\frac{2 i-1}{2 n} \pi, \quad(i=1,2, \cdots, n),
$$

and $T_{n}{ }^{\prime}(\cos \theta)=n(\sin n \theta) / \sin \theta$, we get

$$
p_{n}{ }^{\prime}\left(x_{i}\right)=2 T_{n}{ }^{\prime}\left(\cos \theta_{i}\right)=(-1)^{i-1} 2 n / \sin \theta_{i},
$$

and so,

$$
\left(1+x_{i}\right)\left|p_{n}{ }^{\prime}\left(x_{i}\right)\right|=\frac{3+\cos \theta_{i}}{\sin \theta_{i}} n .
$$

The function $f(\theta)=(3+\cos \theta) / \sin \theta$ has a unique minimum in the interval $(0, \pi)$ which is assumed at $\theta=\theta_{0}$, where $\cos \theta_{0}=-1 / 3$, i.e. $\theta_{0} \doteq \pi / 2+.340$. Let $i=i_{0}$ be such that $\pi / 2 \leqq \theta_{i_{0}}<\theta_{0}$. (The existence of $i_{0}$ is trivial if $n$ is odd, and if $n$ is even is assured whenever $n>4$.) Since $f\left(\theta_{i_{0}}\right) \leqq f(\pi / 2)=3$, we obtain

$$
\left(1+x_{i_{0}}\right)\left|p_{n}{ }^{\prime}\left(x_{i_{0}}\right)\right| \leqq 3 n,
$$

and thus, by (2.5) and (7.1),

$$
\operatorname{cond}_{\infty}\left[V\left(p_{n}{ }^{(\alpha, \beta)}\right)\right]>\frac{1}{6}(3+\sqrt{ } 8)^{n}, \quad\left(\alpha=\beta=-\frac{1}{2}\right),
$$

in agreement with the case $\sigma=0$ of (6.4).

Consider, next, $\alpha=\beta=\frac{1}{2}$, i.e. $p_{n}(x)=U_{n}(2 x-1)$. Here we have

$$
\left|p_{n}(-1)\right|=U_{n}(3)=\frac{(3+\sqrt{ } 8)^{n+1}}{2 \sqrt{ } 8}\left\{1-(17+6 \sqrt{ } 8)^{-n-1}\right\},
$$

and

$$
2 x_{i}-1=\cos \theta_{i}, \quad \theta_{i}=\frac{i}{n+1} \pi, \quad(i=1,2, \cdots, n) .
$$

Since now

$$
U_{n}^{\prime}(\cos \theta)=\frac{1}{\sin ^{3} \theta}[\cos \theta \sin (n+1) \theta-(n+1) \sin \theta \cos (n+1) \theta],
$$

we get

$$
p_{n}^{\prime}\left(x_{i}\right)=2 U_{n}^{\prime}\left(\cos \theta_{i}\right)=(-1)^{i+1} \frac{2(n+1)}{\sin ^{2} \theta_{i}},
$$

and so,

$$
\left(1+x_{i}\right)\left|p_{n}{ }^{\prime}\left(x_{i}\right)\right|=\frac{3+\cos \theta_{i}}{\sin ^{2} \theta_{i}}(n+1) .
$$

In the interval $(0, \pi)$ the function $g(\theta)=(3+\cos \theta) / \sin ^{2} \theta$ takes on its unique minimum at $\theta=\theta_{0}$, where $\cos \theta_{0}=\sqrt{ } 8-3$, i.e. $\theta_{0} \doteq \pi / 2+.173$. Picking $i=i_{0}$ such that $\pi / 2 \leqq \theta_{i_{0}}<\theta_{0}$ (which is always possible if $n$ is odd, and if $n$ is even certainly for $n>8$ ), we have $g\left(\theta_{i_{0}}\right) \leqq g(\pi / 2)=3$, and therefore 


$$
\left(1+x_{i_{0}}\right)\left|p_{n}{ }^{\prime}\left(x_{i_{0}}\right)\right| \leqq 3(n+1) .
$$

Consequently, by (2.5) and (7.3),

$$
\text { cond } \begin{aligned}
{\left[V\left(p_{n}{ }^{(\alpha, \beta)}\right)\right] } & \geqq \frac{n}{(6 \sqrt{ } 8)(n+1)}(3+\sqrt{ } 8)^{n+1}\left\{1-(17+6 \sqrt{ } 8)^{-n-1}\right\} \\
& \sim \frac{(3+\sqrt{ } 8)^{n+1}}{6 \sqrt{ } 8}, \quad\left(\alpha=\beta=\frac{1}{2}, n \rightarrow \infty\right),
\end{aligned}
$$

in agreement with the case $\sigma=1$ of (6.4).

8. For comparison we briefly discuss the case of equidistant abscissas**

$$
x_{i}=i /(n+1), \quad(i=1,2, \cdots, n) .
$$

Here, (2.3) and (2.4) give

$$
\operatorname{cond}_{\infty}\left[V\left(p_{n}\right)\right]=\frac{n(n+2)(n+3) \cdots(2 n+1)}{\min _{i} \pi_{i}},
$$

where

$$
\pi_{i}=(i+n+1) \prod_{j=1 ; j \neq i}^{n}|i-j|, \quad(i=1,2, \cdots, n) .
$$

Observing that

$$
\pi_{i+1}=\frac{i+n+2}{i+n+1} \frac{i}{n-i} \pi_{i}, \quad(i=1,2, \cdots, n-1),
$$

and that the function $f(x)=(x+n+2) x /((x+n+1)(n-x))$ is monotonically increasing on the interval [1,n-1], with $f(1)<1$ (for $n \geqq 3$ ), $f(n-1)>1$ (for $n \geqq 2), f(n / 2)>1, f((n-1) / 2)<1$, it follows that

$$
\pi_{i+1}<\pi_{i} \quad \text { for } i \leqq[(n-1) / 2], \quad \pi_{i+1}>\pi_{i} \quad \text { for } i>[(n-1) / 2] .
$$

Consequently, the minimum in (8.2) occurs at $i=[(n-1) / 2]+1=[(n+1) / 2]$, and we find that

$$
\begin{aligned}
& \operatorname{cond}_{\infty}\left[V\left(p_{n}\right)\right]=\frac{n^{2}}{(3 n+2)(n+1)} \frac{(2 n+1) !}{n !(n / 2) !^{2}}, \quad(n \text { even }), \\
& \operatorname{cond}_{\infty}\left[V\left(p_{n}\right)\right]=\frac{2 n}{3(n+1)} \frac{(2 n+1) !}{(n+1) !((n-1) / 2) !^{2}}, \quad(n \text { odd }) .
\end{aligned}
$$

Therefore, by Stirling's formula,

$$
\operatorname{cond}_{\infty}\left[V\left(p_{n}\right)\right] \sim \frac{2 \sqrt{ } 2}{3 \pi} 8^{n}, \quad(n \rightarrow \infty) .
$$

Department of Computer Sciences

Purdue University

Lafayette, Indiana 47907

** Consideration of this case was suggested to the author by Professor C. H. Wilcox during a recent conversation. 
1. A. B. BAKušinskiř, "On a numerical method for the solution of Fredholm integral equations of the first kind," Z. Vyčisl. Mat. $i$ Mat. Fiz., v. 5, 1965, pp. 744-749. (Russian)

2. A. B. BAKUŠ́nsKiř, "On a certain numerical method of solution of Fredholm integral equations of the first kind," Comput. Methods Programming. Vol. V, Izdat. Moskov. Univ., Moscow, 1966, pp. 99-106. (Russian) MR 35 \#6386.

3. R. Bellman, R. Kalaba \& J. LocketT, "Dynamic programming and ill-conditioned linear systems. II," J. Math. Anal. Appl., v. 12, 1965, pp. 393-400. MR 32 \#9104.

4. R: Bellman, R. E. Kalaba \& J. A. Lockett, Numerical Inversion of the Laplace Transform. Applications to Biology, Economics, Engineering and Physics, American Elsevier, New York, 1966. MR 34 \#5282.

5. W. GAUTSCHI, "On inverses of Vandermonde and confluent Vandermonde matrices," Numer. Math., v. 4, 1962, pp. 117-123. MR $25 \# 3059$.

6. W. GAUTSCHI, "Construction of Gauss-Christoffel quadrature formulas," Math. Comp., v. 22,1968 , pp. 251-270.

7. V. I. KRYLOv, V. V. Lugin \& L. A. JANovič, Tables for the Numerical Integration of Functions with Power Singularities $\int_{0}^{1} x^{\beta}(1-x)^{\alpha} f(x) d x$, Izdat. Akad. Nauk. Belorussk. SSR, Minsk, 1963. (Russian) MR 28 \#253.

8. Y. L. LuKe, Review 6, Math. Comp., v. 22, 1968, pp. 215-218.

9. D. L. PHILLIPS, "A technique for the numerical solution of certain integral equations of the first kind," J. Assoc. Comput. Mach., v. 9, 1962, pp. 84-97. MR 24 \#B534.

10. G. Szegö, Orthogonal Polynomials, Amer. Math. Soc. Colloq. Publ., Vol. 23, Amer. Math. Soc., Providence, R. I., 1959. MR 21 \#5029.

11. A. N. Tinonov \& V. B. Glasko, "An approximate solution of Fredholm integral equations of the first kind," Z. Vyčisl. Mat. $i$ Mat. Fiz., v. 4, 1964, pp. 564-571. (Russian) MR $29 \# 6654$.

12. J. Tod, Introduction to the Constructive Theory of Functions, Academic Press, New York, 1963. MR $27 \# 6061$.

13. S. TwOMEY, "On the numerical solution of Fredholm integral equations of the first kind by the inversion of the linear system produced by quadrature," J. Assoc. Comput. Mach., v.10, 1963, pp. 97-101. MR $29 \# 6655$.

14. P. N. ZAIKIN, "On the numerical solution of the inversion problem of operational calculus in the real domain," Ž. Vyčisl. Mat. i Mat. Fiz., v. 8, 1968, pp. 411-415. (Russian) 